\title{
Temporal validation of metabolic nodal response of esophageal cancer to neoadjuvant chemotherapy as an independent predictor of unresectable disease, survival, and recurrence
}

\author{
John M. Findlay ${ }^{1,2}$ (D) $\cdot$ Edward Dickson ${ }^{1} \cdot$ Cristina Fiorani $^{1} \cdot$ Kevin M. Bradley $^{3} \cdot$ Somnath Mukherjee $^{2} \cdot$ \\ Richard S. Gillies ${ }^{1} \cdot$ Nicholas D. Maynard ${ }^{1}$ - Mark R. Middleton ${ }^{2}$
}

Received: 15 February 2019 / Revised: 21 May 2019 / Accepted: 7 June 2019 / Published online: 5 July 2019

(C) The Author(s) 2019

\begin{abstract}
Objectives We recently described metabolic nodal stage $(\mathrm{mN})$ and response $(\mathrm{mNR})$ of cancer of the esophagus and gastroesophageal junction (GEJ) to neoadjuvant chemotherapy (NAC) using ${ }^{18} \mathrm{~F}$-FDG PET-CT as new markers of disease progression, recurrence, and death. We aimed to validate our findings.

Methods Our validation cohort comprised all patients consecutive to our discovery cohort, staged before and after NAC using PET-CT from 2014 to 2017. Multivariate binary logistic and Cox regression were performed.

Results Fifty-one of the 200 patients had FDG-avid nodes after NAC (25.5\%; i.e., lack of complete mNR), and were more likely to progress during NAC to incurable disease on PET-CT or at surgery: odds ratio $3.84(1.46-10.1 ; p=0.006)$. In 176 patients undergoing successful resection, patients without complete $\mathrm{mNR}$ had a worse prognosis: disease-free survival hazard ratio 2.46 (1.34-4.50); $p=0.004$. These associations were independent of primary tumor metabolic, pathological response, and stage. In a hybrid pathological/metabolic nodal stage, avid nodal metastases conferred a worse prognosis than non-avid metastases. Lack of complete $\mathrm{mNR}$ predicted recurrence or death at 1 and 2 years: positive predictive values $44.4 \%$ (31.7-57.8) and $74.1 \%$ (56.6-86.3) respectively. Conclusions This study provides temporal validation for $\mathrm{mNR}$ as a new and independent predictive and prognostic marker of esophageal and GEJ cancer treated with NAC and surgery, although external validation is required to assess generalizability. $\mathrm{mNR}$ may provide surrogate information regarding the phenotype of metastatic cancer clones beyond the mere presence of nodal metastases, and might be used to better inform patients, risk stratify, and personalize management, including adjuvant therapy. Key Points

- We previously described metabolic nodal response (mNR) of esophageal cancer to neoadjuvant chemotherapy using ${ }^{18} F-F D G$ PET-CT as a predictor of unresectable disease, early recurrence, and death.

- We report the first validation of these findings. In an immediately consecutive cohort, we found consistent proportions of patients with and without $m N R$, and associations with abandoned resection, early recurrence, and death.

- This supports $m N R$ as a new and actionable biomarker in esophageal cancer. Although external validation is required, $m N R$ may provide surrogate information about the chemosensitivity of metastatic subclones, and the means to predict treatment success, guide personalized therapy, and follow-up.
\end{abstract}

Keywords Esophagectomy $\cdot$ Prognosis $\cdot$ Recurrence $\cdot$ PET-CT $\cdot$ Metastases

Electronic supplementary material The online version of this article (https://doi.org/10.1007/s00330-019-06310-9) contains supplementary material, which is available to authorized users.

John M. Findlay

john.findlay@oncology.ox.ac.uk

1 Oxford Oesophagogastric Centre, Churchill Hospital, Oxford OX3 7LE, UK
Department of Oncology, Old Road Campus Research Building, University of Oxford, Oxford OX3 7DQ, UK

3 Department of Nuclear Medicine, Churchill Hospital, Oxford OX3 7LE, UK 


$\begin{array}{ll}\text { Abbreviations } \\ \text { BPL } & \text { Bayesian penalized likelihood } \\ \text { CI } & \text { Confidence interval } \\ \text { CMR } & \text { Complete metabolic response } \\ \text { CT } & \text { Computed tomography } \\ \text { DFS } & \text { Disease-free survival } \\ \text { EUS } & \text { Endoscopic ultrasound } \\ \text { FDG } & { }^{18} \text { F-fluorodeoxyglucose } \\ \text { GE } & \text { General Electric } \\ \text { GEJ } & \text { Gastro-esophageal junction } \\ \text { MDT } & \text { Multidisciplinary team } \\ \text { mN } & \text { Metabolic nodal stage } \\ \text { mNR } & \text { Metabolic nodal response } \\ \text { NPV } & \text { Negative predictive value } \\ \text { mTR } & \text { Metabolic tumor response } \\ \text { NAC } & \text { Neoadjuvant chemotherapy } \\ \text { NHS } & \text { National Health Service } \\ \text { OS } & \text { Overall survival } \\ \text { OSEM } & \text { Ordered subset expectation maximization } \\ \text { PERCIST } & \text { Positron Emission Tomography Response } \\ & \text { Criteria in Solid Tumors } \\ \text { PET-CT } & \text { Positron emission tomography-computed } \\ & \text { tomography } \\ \text { PMD } & \text { Progressive metabolic disease } \\ \text { PMR } & \text { Partial metabolic response } \\ \text { PPV } & \text { Positive predictive value } \\ \text { SMD } & \text { Stable metabolic disease } \\ \text { SUVmax } & \text { Maximum standardized uptake value } \\ \text { ypN } & \text { Pathological nodal stage after chemotherapy } \\ \text { ypT } & \text { Pathological tumor stage after chemotherapy } \\ & \end{array}$

\section{Introduction}

We recently reported the new concepts of metabolic nodal stage $(\mathrm{mN})$ and response $(\mathrm{mNR})$ of esophageal and gastroesophageal junction (GEJ) cancer to neoadjuvant chemotherapy (NAC) $[1,2]$. We found that patients with FDGavid nodes within a standard lymphadenectomy field on ${ }^{18} \mathrm{~F}$-fluorodeoxyglucose (FDG) positron emission tomography-computed tomography (PET-CT) that persisted despite NAC (i.e. lacking a complete metabolic nodal response, CMR) were more likely to suffer disease progression during NAC which prevented resection (i.e., distant metastases, or locally unresectable disease). Furthermore, if resection was performed successfully, these patients were more likely to suffer disease recurrence. This was independent of pathological stage, and both pathological and metabolic regression of the primary tumor [1, 2], suggesting mNR to be a surrogate of regression of nodal metastases to NAC. Indeed, in some patients notable disparity was seen between primary and nodal response, suggesting $\mathrm{mNR}$ might represent a surrogate of the chemosensitivity of different clones, and perhaps by extrapolation occult distant metastatic clones.

mNR therefore represents a potentially novel and actionable biomarker of the success both of NAC and radical lymphadenectomy, for which there is an urgent need [3-6]. Such a marker might have the potential to monitor response to NAC and perhaps personalize therapy, while also better prognosticating before surgery, and stratifying the risk of recurrence thereafter. To our knowledge, no studies have subsequently assessed $\mathrm{mNR}$ as a marker of disease progression and recurrence. We aimed to perform the first validation study of $\mathrm{mNR}$ as a marker of disease progression and recurrence, using a temporal cohort of patients immediately consecutive to our discovery cohort.

\section{Methods}

\section{Study design and approval}

We routinely stage and restage patients with esophageal or GEJ cancer using ${ }^{18} \mathrm{~F}$-FDG PET-CT, and retrospectively identified all such patients receiving NAC from January 2014 to June 2017. The study was approved by the Oxford University Hospitals NHS Foundation Trust Research and Development committee.

\section{Inclusion and exclusion criteria}

We included all patients with histologically confirmed cancer of the esophagus or GEJ, staged in our institution with ${ }^{18} \mathrm{~F}$ FDG PET-CT, undergoing NAC before being restaged with ${ }^{18}$ F-FDG PET-CT. We excluded patients treated with neoadjuvant chemoradiotherapy, as well as those undergoing PET$\mathrm{CT}$ in other centers.

\section{Staging and restaging}

All examinations were reported by subspecialist consultant gastrointestinal radiologists and reviewed at a specialist esophagogastric cancer multidisciplinary team (MDT) meeting, using the TNM 7 th edition [7]. Patients underwent intravenous and oral contrast-enhanced CT chest abdomen and pelvis, as previously reported $[1,2]$. In the absence of unequivocal incurable disease, patients then underwent PET-CT (see below), followed by laparoscopy (without peritoneal cytology) for tumors extending below the diaphragm. Endoscopic ultrasound (EUS) was performed selectively. Examinations were reported. Patients were restaged 4-6 weeks after NAC.

PET-CT was performed from January 2014 using a General Electric (GE) Discovery 690 64-slice system, then also a GE Discovery 710 from September 2014 (both 90 min post 
$4 \mathrm{MBq} / \mathrm{Kg}$ FDG), all using ordered subset expectation maximization (OSEM) reconstruction; after 1 November 2014, a Bayesian penalized likelihood (BPL) reconstruction technique was used (Q.Clear) without intravenous contrast. Any staging studies initially reconstructed with OSEM and undergoing a subsequent restaging examination with BPL had their baseline studies reconstructed with BPL to ensure validity. Examinations were independently reported by two dedicated PET-CT radiologists, dual trained in nuclear medicine and clinical radiology.

\section{Data}

PET-CT data comprised primary tumor maximum standardized uptake value (SUVmax), the presence and number of FDG-avid nodes ( $\mathrm{mN}$, as previously defined: $\mathrm{mN} 1$ 1-2 avid nodes, mN2 > 2), in both cases, a target SUVmax greater than mediastinal blood pool being avid [1]; restaging SUVmax (used to calculate metabolic tumor response [mTR], \% reduction SUVmax normalized for background SUVmax [2.5], and PERCIST classification [8]); metastatic disease; $\mathrm{mNR}$ as previously defined (complete metabolic response [CMR] to background mediastinal blood pool avidity, partial metabolic response [PMR; reduction in $\mathrm{mN}$ stage or \% reduction in nodal SUVmax $>30 \%$ ], stable metabolic disease [SMD; no change in $\mathrm{mN}$ stage, or nodal SUVmax $<30 \%$ ], and progressive metabolic disease [PMD; progression in $\mathrm{mN}$ stage, or increase nodal SUVmax $>30 \%]$ ).

Pathological data comprised pathological stage [7], and primary tumor regression (Mandard tumor regression grade 1-3 being response, 4-5 no response [2, 9]). Survival data comprised recurrence and death (censored on 23 March 2018). Disease progression to incurable disease during NAC was defined as either metastatic disease on imaging (confirmed by MDT discussion \pm confirmatory imaging/histopathology) or unresectable disease at surgery (confirmed intra-operatively by frozen section histopathology, or consensus of two consultant surgeons).

\section{Neoadjuvant chemotherapy}

Patients received a variety of regimens based on MDT consensus and trial enrolment: either 2 cycles of doublet therapy (cisplatin/oxaliplatin and 5-fluorouracil/capecitabine) or 3 or 4 cycles of pre- \pm post-operative triplet therapy (epirubicin, cisplatin/oxaliplatin and 5-fluorouracil/capecitabine) as previously described [10-14].

\section{Surgery}

Esophagectomy (for esophageal or GEJ type I-II cancers) or extended total gastrectomy (types II-III) was performed typically 2 weeks after restaging, via left thoracoabdominal, Ivor-Lewis (right thoracotomy plus laparotomy/laparoscopy), and three-stage approaches (laparotomy/laparoscopy, thoracotomy plus neck dissection). A minimum two-field lymphadenectomy was performed; for $\mathrm{mid} /$ distal esophageal/type $1 \mathrm{GEJ}$ tumors, this included subcarinal, aortopulmonary window, and left gastric nodes, and for distal/GEJ tumors, left gastric and common hepatic artery nodes.

\section{Follow-up}

Patients were reviewed clinically at 2 and 6 weeks after surgery, 3 months to 1 year and 6-12 months thereafter for at least 5 years. Investigations for recurrence were performed only on the basis of clinical suspicion, typically CT or PET-CT for extra-luminal recurrence, and endoscopy for luminal.

\section{Statistical analysis}

Analysis was performed using R v3.0.2 [15]. Groups were compared using Fisher's exact test. Univariate binary logistic and Cox regression analysis were performed using all individual data fields. Multivariate binary logistic and Cox regression were performed using variables with $p<0.1$ on univariate analysis for inclusion in the final multivariate model. The sole patient with an adenosquamous carcinoma was excluded from univariate analysis of cell type, being a perfect separator. The final multivariate binary logistic regression models for disease progression comprised $\mathrm{mTR}$ and restaging $\mathrm{mN}$; for unresectable disease at surgery, restaging $\mathrm{mN}$ and pretreatment grade of differentiation; and for diseaseprogression overall, restaging $\mathrm{mN}$ and clinical $\mathrm{N}$ stage. The final multivariate Cox models for survival comprised mNR, ypT, ypN, ypV (except for overall survival, as $p>0.1$ on univariate analysis), ypL, resection margin status, pTR, and mTR. For the model assessing a pNmN hybrid, the models were as above, having excluded mNR and ypN due to multicollinearity. The final multivariate binary logistic regression model for early recurrence/death comprised of $\mathrm{mnR}, \mathrm{ypT}, \mathrm{ypN}$, ypL, resection margin status, pTR, and mTR. Similarly, the model assessing a pNmN hybrid excluded mNR and ypN. Survival metrics were calculated using the Kaplan-Meier method, and follow-up using the reverse Kaplan-Meier method, excluding patients dying in hospital due to post-operative complications. Kaplan-Meier curves were generated using survfit (Survival Package v2.42-3) using the log-rank test. 


\section{Results}

\section{$\mathrm{mN}$ stage and response}

Two hundred patients were included (Table 1). Eighty-one $(40.5 \%)$ had avid nodes before NAC. Thirty-eight (46.9\%) underwent CMR. Eight (4.00\% overall) previously mN0 patients developed avid nodes. In total, 51 patients $(25.5 \%)$ had avid nodes following NAC. This was consistent with the development cohort: 64/280 (22.9\%; $p=0.617)$. PET reconstruction algorithms did not influence mNR, mTR, and detection of metastatic disease (Supplementary Table 1).

\section{Progression to incurable disease on restaging PET-CT}

Incurable disease was identified in $10(5.00 \%)$ patients. No pre-treatment factors were associated with this. On multivariate binary logistic regression, both the presence of avid nodes at restaging ( $\mathrm{mN}$ stage 2$)$ and lack of $\mathrm{mTR}$ (\% reduction in primary tumor SUVmax) were associated with coexistent metastatic disease (Table 2). Five of $51(9.80 \%)$ patients with avid nodes after NAC had metastatic disease, compared with 5 of $149(3.36 \%)$ without $(p=0.127)$.

\section{Progression to unresectable disease at surgery}

A further 2 patients without metastases evident on restaging PET-CT did not proceed to surgery for medical reasons (both had avid nodes). One hundred eighty-eight proceeded to surgery. Unsuspected unresectable disease was encountered in 10 $(5.32 \%)$. On multivariate analysis, this was associated with the presence of avid nodes following NAC: OR 7.67 (1.94 $30.4 ; p=0.004$; Table 2).

\section{Disease progression overall}

Overall, avid nodes following NAC predicted disease progression: OR $3.84(1.46-10.1 ; p=0.006)$, independent of all variables including clinical $\mathrm{N}$ stage. As with progression on PET$\mathrm{CT}$, this association was primarily seen for patients with $\mathrm{mN} 2$ disease after NAC.

\section{Predicting unresectable disease at surgery}

Before surgery, 44 of 188 patients (23.4\%) had avid nodes following NAC. Of these, $6(14.0 \%)$ had an abandoned resection, compared with 4 of 144 without $(2.78 \% ; p=0.011 ; 6$ true positives, 38 false positives, 4 false negatives, and 140 true negatives) The presence of avid nodes despite NAC was $60.0 \%$ sensitive $(26.2-87.8)$ and $78.7 \%$ specific (71.9-84.4) for predicting unsuspected unresectable disease. Positive predictive value (PPV) was $13.6 \%$ (8.13-22.0), and negative predictive value (NPV) was $97.2 \%$ (94.2-98.9\%).
Table 1 Patients

\begin{tabular}{|c|c|c|}
\hline Characteristic & $\begin{array}{l}\text { All patients } \\
(n=200)\end{array}$ & $\begin{array}{l}\text { Survival analysis } \\
(n=176)\end{array}$ \\
\hline Age (median, IQR) & $70.0(63.0-74.0)$ & $70.0(63.0-74.0)$ \\
\hline \multicolumn{3}{|l|}{ Gender } \\
\hline Male & $159(79.5 \%)$ & $137(77.8 \%)$ \\
\hline Female & $41(20.5 \%)$ & $39(22.2 \%)$ \\
\hline \multicolumn{3}{|l|}{ Tumor cell type } \\
\hline $\mathrm{AC}$ & $183(91.5 \%)$ & $161(91.5 \%)$ \\
\hline SCC & $16(8.00 \%)$ & $14(7.95 \%)$ \\
\hline AS & $1(0.50 \%)$ & $1(0.58 . \%)$ \\
\hline \multicolumn{3}{|l|}{ Grade of differentiation } \\
\hline Well & $4(2.00 \%)$ & $3(1.70 \%)$ \\
\hline Moderate & $93(46.5 \%)$ & $86(48.9 \%)$ \\
\hline Poor/undifferentiated & $103(51.5 \%)$ & $87(49.4 \%)$ \\
\hline \multicolumn{3}{|l|}{ Tumor site } \\
\hline Mid esophageal & $15(7.50 \%)$ & $12(6.82 \%)$ \\
\hline Distal esophageal & $77(38.5 \%)$ & $67(38.1 \%)$ \\
\hline GEJ1 & $55(27.5 \%)$ & $53(29.5 \%)$ \\
\hline GEJ2 & $29(14.5 \%)$ & $25(14.2 \%)$ \\
\hline GEJ3 & $24(12.0 \%)$ & $19(10.8 \%)$ \\
\hline \multicolumn{3}{|l|}{ Pre-chemotherapy $\mathrm{mN}$ stage } \\
\hline 0 & $119(59.5 \%)$ & $108(61.4 \%)$ \\
\hline 1 & $47(23.5 \%)$ & $40(22.7 \%)$ \\
\hline 2 & $34(17.0 \%)$ & $28(15.9 \%)$ \\
\hline \multicolumn{3}{|c|}{ Metabolic nodal response (mNR) } \\
\hline No avid nodes & $111(55.5 \%)$ & $102(58.0 \%)$ \\
\hline CMR & $38(19.0 \%)$ & $35(19.9 \%)$ \\
\hline PMR & $9(4.5 \%)$ & $8(4.54 \%)$ \\
\hline SMD & $25(12.5 \%)$ & $20(11.4 \%)$ \\
\hline PMD & $9(4.5 \%)$ & $6(3.41 \%)$ \\
\hline de novo avid nodes & $8(4.00 \%)$ & $5(2.84 \%)$ \\
\hline \multicolumn{3}{|l|}{ mTR } \\
\hline $\begin{array}{l}\text { \% Reduction SUVmax } \\
\text { (median, IQR) }\end{array}$ & $\begin{array}{l}42.4 \% \\
\quad(12.8-60.6 \%)\end{array}$ & $45.5 \%(15.8-62.9 \%)$ \\
\hline CMR & $15(7.50 \%)$ & $15(8.53 \%)$ \\
\hline $\begin{array}{l}\text { T stage (clinical or } \\
\text { pathological) }\end{array}$ & Clinical T stage & $\begin{array}{l}\text { Pathological T stage, } \\
\text { урT }\end{array}$ \\
\hline $1 b$ & $5(2.50 \%)$ & $29(16.5 \%)$ \\
\hline 2 & $34(17.0 \%)$ & $20(11.4 \%)$ \\
\hline 3 & $133(66.5 \%)$ & $116(65.9 \%)$ \\
\hline 4 & $28(14.0 \%)$ & $11(6.25 \%)$ \\
\hline $\begin{array}{l}\mathrm{N} \text { stage (clinical or } \\
\text { pathological) }\end{array}$ & Clinical N stage & $\begin{array}{l}\text { Pathological N stage, } \\
\text { ypN }\end{array}$ \\
\hline 0 & $64(32.0 \%)$ & $67(38.1 \%)$ \\
\hline 1 & $83(41.5 \%)$ & $43(24.4 \%)$ \\
\hline 2 & $47(23.5 \%)$ & $38(21.6 \%)$ \\
\hline 3 & $6(3.00 \%)$ & $28(15.9 \%)$ \\
\hline ypV & NA & \\
\hline 0 & & $102(58.0 \%)$ \\
\hline 1 & & $73(41.5 \%)$ \\
\hline Not known & & 1 \\
\hline
\end{tabular}


Table 1 (continued)

\begin{tabular}{llc}
\hline Characteristic & $\begin{array}{l}\text { All patients } \\
(n=200)\end{array}$ & $\begin{array}{l}\text { Survival analysis } \\
(n=176)\end{array}$ \\
\hline ypL & NA & $101(57.4 \%)$ \\
0 & & $74(42.0 \%)$ \\
1 & 1 \\
Not known & $147(83.5 \%)$ \\
Resection margin status & $29(16.5 \%)$ \\
Clear & \\
Involved & $137(49.6 \%)$ \\
pTR & $38(21.6 \%)$ \\
No response & 1 \\
Response & \\
Not known & \\
\hline
\end{tabular}

$A C$, adenocarcinoma; $S C C$, squamous cell carcinoma; $A S$, adenosquamous carcinoma (excluded from multivariate analysis as perfect separator); $I Q R$, interquartile range; $m T R$, metabolic tumor response; $C M R$, complete metabolic response; $P M R$, partial metabolic response; $S M D$, stable metabolic disease; $P M D$, progressive metabolic disease; $y p T$, pathological tumor stage after chemotherapy; $y p N$, pathological nodal stage; $y p V$, pathological venous invasion; $y p L$, pathological lymphatic invasion; $p T R$, pathological tumor response

These proportions were less than the development cohort, in which 11 of $43(25.6 \%)$ patients with avid nodes had an abandoned resection, compared with 15 of 197 (7.61\%) without. Rates of abandoned resections halved from 10.5 to $5.32 \%$.

\section{Prognosis}

Two patients (1.06\%) died in hospital of post-operative complications and were excluded. Of the remaining 176 patients (Table 1), 55 died (31.3\%) and 59 (33.5\%) developed recurrence during follow-up. Median OS, DFS, and recurrence were not reached (respective 95\% CI 1067 days - not reached; 770 - not reached; 872 - not reached). Median follow-up was 783 days (737-886).

On multivariate Cox analysis, the presence of avid nodes following NAC was associated with death and recurrence following resection: OS HR $2.46(1.34-4.51 ; p=0.004)$, DFS HR $1.90(1.10-3.31 ; p=0.022)$, and time to recurrence HR 2.02 (1.11-3.66; $p=0.021$; Table 3; Fig. 1). This was independent of similar negative associations with progressive ypN stage. No other factors, including mTR (either reduction in SUVmax, or PERCIST), were independently associated with prognosis. There were no clinically or statistically significant differences when $\mathrm{mNR}$ was quantified using $\mathrm{mN}$ stage after NAC (i.e., the number of avid nodes). These HR were similar to the development cohort: OS $1.75(0.99-3.07 ; p=0.053)$, DFS $2.03(1.16-3.55 ; p=0.013)$, and recurrence $2.06(1.10$ $3.83 ; p=0.023)$.

\section{Hybrid pathological metabolic nodal stage}

As both $\mathrm{mNR}$ and ypN independently predicted prognosis, we considered a composite. Overall 109 patients had nodal metastases (ypN+), while 38 had avid nodes $(\mathrm{mN}+)$. Considering post-NAC $\mathrm{mN}$, a predictor of $\mathrm{ypN}$, there were 31 true positives, 7 false positives, 78 false negatives, and 60 true negatives. $\mathrm{mNR}$ was $28.4 \%$ sensitive (20.2-37.9), and $89.5 \%$ specific (79.7-95.7), PPV 81.6\% (76.4-90.5), and NPV $43.5 \%$ (40.0-47.0).

This ypNmN stage was associated with prognosis (Table 3 ; Fig. 2): relative to $\mathrm{pN} 0 \mathrm{mN} 0$ disease, patients with non-avid nodal metastases $(\mathrm{ypN}+\mathrm{mN}-$ ) had a worse prognosis. However, prognosis was even worse for patients with avid nodal metastases ( $\mathrm{ypN}+\mathrm{mN}+$ ). Interestingly, the small number with ypN-mN+ disease (i.e., presumed false avid positive) demonstrated a trend towards worse prognosis for DFS $(p=0.085)$ with significantly worse recurrence, suggesting the possibility of pathologically missed micro-metastases.

\section{Predicting early recurrence and death}

One hundred twenty-nine of $161(78.2 \%)$ patients were alive and disease-free at 1 year, reducing to 68 of $128(53.1 \%)$ at 2 years. On multivariate regression, lack of complete mNR was associated with recurrence and death at both time points: OR $4.18(1.56-11.2 ; p=0.004)$ and $3.63(1.26-10.5$; $p=0.017)$ respectively. This was again independent of associations with ypN (Table 4). The associations with ypN were primarily for recurrence/death within 1 year, rather than 2 years. At 2 years, recurrence and death were associated with hybrid $\mathrm{pNmN}$ stage.

At 1 year, 16 of 36 (44.4\%) patients with persistent avid nodes had either died or developed recurrence, compared with 20 of $129(15.5 \%)$ without ( $p<0.001$ Fisher's exact test). Lack of a nodal CMR was $44.4 \%$ sensitive (27.9-61.9) and 84.5\% specific (77.1-90.3), with PPV 44.4\% (31.7-57.8) and NPV 84.5 (80.1-88.1).

At 2 years, 20 of 27 (74.1\%) patients with persistent avid nodes had either died or developed recurrence, compared with 38 of 99 without (38.4\%; $p=0.001)$. Lack of nodal CMR was $34.5 \%$ sensitive (22.5-48.1) and 89.7\% specific (79.9-95.8), with PPV 74.1\% (56.6-86.3) and NPV 61.6\% (56.7-66.3).

\section{Discussion}

We previously reported lack of complete metabolic nodal response of esophageal cancer to NAC to be a new and independent predictor of disease progression to unresectable disease [1], death, and recurrence [2]. In this validation study of an immediately consecutive cohort of 200 patients, we confirmed these associations and were able to risk stratify 
Table 2 Disease progression: multivariate binary logistic regression

\begin{tabular}{|c|c|c|}
\hline Characteristic & $\begin{array}{l}\text { Odds ratio (OR; } 95 \% \\
\text { confidence interval) }\end{array}$ & $p$ \\
\hline \multicolumn{3}{|c|}{ Progression to overt metastatic disease on PET-CT } \\
\hline \multicolumn{3}{|l|}{ Restaging $\mathrm{mN}$} \\
\hline mN0 & Ref & \\
\hline $\mathrm{mN1}$ & $0.78(0.08-7.38)$ & 0.832 \\
\hline $\mathrm{mN} 2$ & $4.37(1.03-18.6)$ & 0.045 \\
\hline \multicolumn{3}{|l|}{ mTR } \\
\hline$\%$ reduction in SUVmax & $0.11(0.02-0.56)$ & 0.008 \\
\hline \multicolumn{3}{|c|}{ Progression to unsuspected unresectable disease at surgery } \\
\hline \multicolumn{3}{|l|}{ Restaging $\mathrm{mN}$} \\
\hline $\mathrm{mN} 0$ & Ref & \\
\hline $\mathrm{mN} 1$ & $6.25(1.24-31.6)$ & 0.027 \\
\hline $\mathrm{mN} 2$ & $8.28(1.59-43.2)$ & 0.012 \\
\hline \multicolumn{3}{|l|}{ Grade } \\
\hline Well/moderate & Ref & \\
\hline Poor & $5.85(1.14-30.0)$ & 0.034 \\
\hline \multicolumn{3}{|l|}{ FDG-avid nodes after NAC } \\
\hline No & Ref & \\
\hline Yes & $7.67(1.94-30.4)$ & 0.004 \\
\hline \multicolumn{3}{|l|}{ Grade } \\
\hline Well/moderate & Ref & \\
\hline Poor & $0.17(0.04-0.91)$ & 0.038 \\
\hline \multicolumn{3}{|l|}{ Disease progression overall } \\
\hline \multicolumn{3}{|l|}{ Restaging $\mathrm{mN}$} \\
\hline mN0 & Ref & \\
\hline $\mathrm{mN} 1$ & $2.79(0.78-10.0)$ & 0.114 \\
\hline $\mathrm{mN} 2$ & $4.62(1.48-14.4)$ & 0.001 \\
\hline \multicolumn{3}{|l|}{ Staging N } \\
\hline 0 & Ref & Ref \\
\hline $1+$ & $3.43(0.0 .73-16.1)$ & 0.118 \\
\hline \multicolumn{3}{|l|}{ FDG-avid nodes after NAC } \\
\hline No & Ref & \\
\hline Yes & $3.84(1.46-10.1)$ & 0.006 \\
\hline \multicolumn{3}{|l|}{ Staging N } \\
\hline 0 & Ref & \\
\hline $1+$ & $3.79(0.83-17.2)$ & 0.085 \\
\hline
\end{tabular}

$m N$, metabolic nodal stage; $N A C$, neoadjuvant chemotherapy; $m T R$, metabolic tumor response; $F D G$, fluorodeoxyglucose

recurrence and death within 1 and 2 years. Compared with our development cohort, effect sizes were entirely consistent for prognosis. That for unresectable disease while significant and consistent, was less clear, reflective of a reduction in event rate overall with time.

These findings have a number of implications. Most immediately, $\mathrm{mNR}$ appears to a simple way to risk stratify patients before surgery, firstly identifying patients at higher risk of occult disease resulting in an abandoned resection. Guidelines presently recommend restaging with $\mathrm{CT}$ to exclude disease progression before surgery [16]. We previously reported PET-CT to be more sensitive [1]; however, this still failed to identify incurable disease in 5\% of our patients. Identifying lack of complete mNR might allow these patients to undergo additional investigations (such as laparoscopy, thoracoscopy, or magnetic resonance imaging). These patients may also benefit from more personalized and realistic information about their immediate risk of an abandoned resection, and medium- and long-term prognosis if 
Table 3 Prognosis: multivariate Cox regression analysis

\begin{tabular}{|c|c|c|c|}
\hline \multirow[t]{2}{*}{ Characteristic } & \multicolumn{3}{|c|}{ Hazard ratio (HR; 95\% confidence interval); $p$ value } \\
\hline & Overall survival & Disease-free survival & Time to recurrence \\
\hline \multicolumn{4}{|l|}{$\mathrm{mNR}$} \\
\hline No avid nodes/CMR & Reference & Reference & Reference \\
\hline $\mathrm{PMR} / \mathrm{SMD} / \mathrm{PMD}$ & $2.46(1.34-4.51 ; 0.004)$ & $1.90(1.10-3.31 ; 0.022)$ & $2.02(1.11-3.66 ; 0.021)$ \\
\hline \multicolumn{4}{|l|}{ ypT stage } \\
\hline $1 \mathrm{~b}$ & Reference & Reference & Reference \\
\hline 2 & $0.51(0.09-2.91 ; 0.449)$ & $0.83(0.21-3.21 ; 0.784)$ & $0.96(0.23-4.04 ; 0.953)$ \\
\hline 3 & $0.75(0.22-2.61 ; 0.656)$ & $0.83(0.28-2.46 ; 0.739)$ & $0.80(0.24-2.66 ; 0.720)$ \\
\hline 4 & $1.45(0.34-6.49 ; 0.594)$ & $1.69(0.46-5.22 ; 0.429)$ & $1.62(0.39-6.38 ; 0.509)$ \\
\hline \multicolumn{4}{|l|}{ ypN stage } \\
\hline 0 & Reference & Reference & Reference \\
\hline 1 & $2.19(0.81-5.95 ; 0123)$ & $3.04(1.26-7.32 ; 0.013)$ & $3.19(1.19-8.54 ; 0.021)$ \\
\hline 2 & $2.88(1.07-7.74 ; 0.036)$ & $3.78(1.54-9.26 ; 0.004)$ & $4.45(1.66-12.1 ; 0.003)$ \\
\hline 3 & $3.81(1.34-10.8 ; 0.012)$ & $4.62(1.81-11.8 ; 0.001)$ & $4.21(1.47-12.1 ; 0.007)$ \\
\hline \multicolumn{4}{|l|}{ ypV stage } \\
\hline 0 & Not included as univariate $p>0.1$ & Reference & Reference \\
\hline 1 & & $0.90(0.50-1.61 ; 0.715)$ & $0.83(0.44-1.57 ; 0.572)$ \\
\hline \multicolumn{4}{|l|}{ ypL stage } \\
\hline 0 & Reference & Reference & Reference \\
\hline 1 & $1.34(059-3.06 ; 0.488)$ & $1.54(0.72-3.29 ; 0.264)$ & $1.80(0.78-4.15 ; 0.167)$ \\
\hline \multicolumn{4}{|l|}{ Resection margin } \\
\hline Clear & Reference & Reference & Reference \\
\hline Involved & $2.00(0.97-4.13 ; 0.062)$ & $1.79(0.95-3.38 ; 0.073)$ & $1.43(0.69-2.93 ; 0.333)$ \\
\hline \multicolumn{4}{|l|}{ pTR } \\
\hline No response & Reference & Reference & Reference \\
\hline Response & $0.69(0.19-1.90 ; 0.390)$ & $0.79(0.32-1.94 ; 0.610)$ & $0.83(0.32-2.21 ; 0.715)$ \\
\hline \multicolumn{4}{|l|}{ mTR } \\
\hline \%Reduction SUVmax & $0.79(0.33-1.86 ; 0.584)$ & $0.88(0.40-1.92 ; 0.753$ & $0.77(0.34-1.76 ; 0.536)$ \\
\hline
\end{tabular}

$\mathrm{pN}$ Mn hybrid, adjusted for variables above other than $\mathrm{pN}$ and $\mathrm{mNR}$ $\mathrm{pN} \mathrm{mN}$ hybrid

$\begin{array}{ll}\mathrm{pN} 0 \mathrm{mN} 0 & \text { Reference } \\ \mathrm{pN}-\mathrm{mN}+ & 1.91(0.23-15.6 ; 0.546) \\ \mathrm{pN}+\mathrm{mN}- & 3.87(1.68-8.89 ; 0.001) \\ \mathrm{pN}+\mathrm{mN}+ & 10.5(4.37-25.2 ;<0.001)\end{array}$

Reference

$4.00(0.83-19.4 ; 0.085)$

$5.75(2.75-12.9 ;<0.001)$

$11.1(4.68-26.1 ;<0.001)$
Reference

$5.60(1.08-29.1 ; 0.040)$

$6.86(2.68-17.6 ;<0.001)$

$13.5(5.00-36.5 ;<0.001)$

$C M R$, complete metabolic response; $P M R$, partial metabolic response; $S M D$, stable metabolic disease; $P M D$, progressive metabolic disease; $m N R$, metabolic nodal response; $m T R$, metabolic tumor response; $y p T$, pathological tumor stage after NAC; $y p N$, pathological nodal stage; $y p V$, pathological venous stage; $y p L$, pathological venous stage; $p T R$, pathological tumor response

surgery is successful. While this may not routinely alter practice - other than for patients unclear as to whether to proceed with surgery - patients can receive more realistic information before consenting to surgery. While there is little evidence or consensus as to follow-up, mNR might be incorporated into decision support tools for targeted surveillance, and future editions of the TNM classification. Finally, the suggestion that patients with avid nodal disease, seemingly in the absence of pathological nodal disease, have a worse prognosis than those without either is notable. Such instances might be dismissed as false positives on PET-CT, but the converse might be true, with these instances identifying occult but viable nodal micrometastases.

Biologically, this statistical independence of $\mathrm{pN}$ and $\mathrm{mNR}$ is intriguing. As evidenced by the prognostic significance of a novel $\mathrm{pNmN}$ hybrid stage, avid nodal metastases confer a worse prognosis than non-avid metastases. This suggests persistent nodal avidity to be a surrogate of a worse cancer phenotype. While the reasons for nodal avidity are complex and incompletely understood (perhaps reflecting mutational burden disrupting glucose utilization, in conjunction with tumor 
Disease-free survival and mNR after neoadjuvant chemotherapy

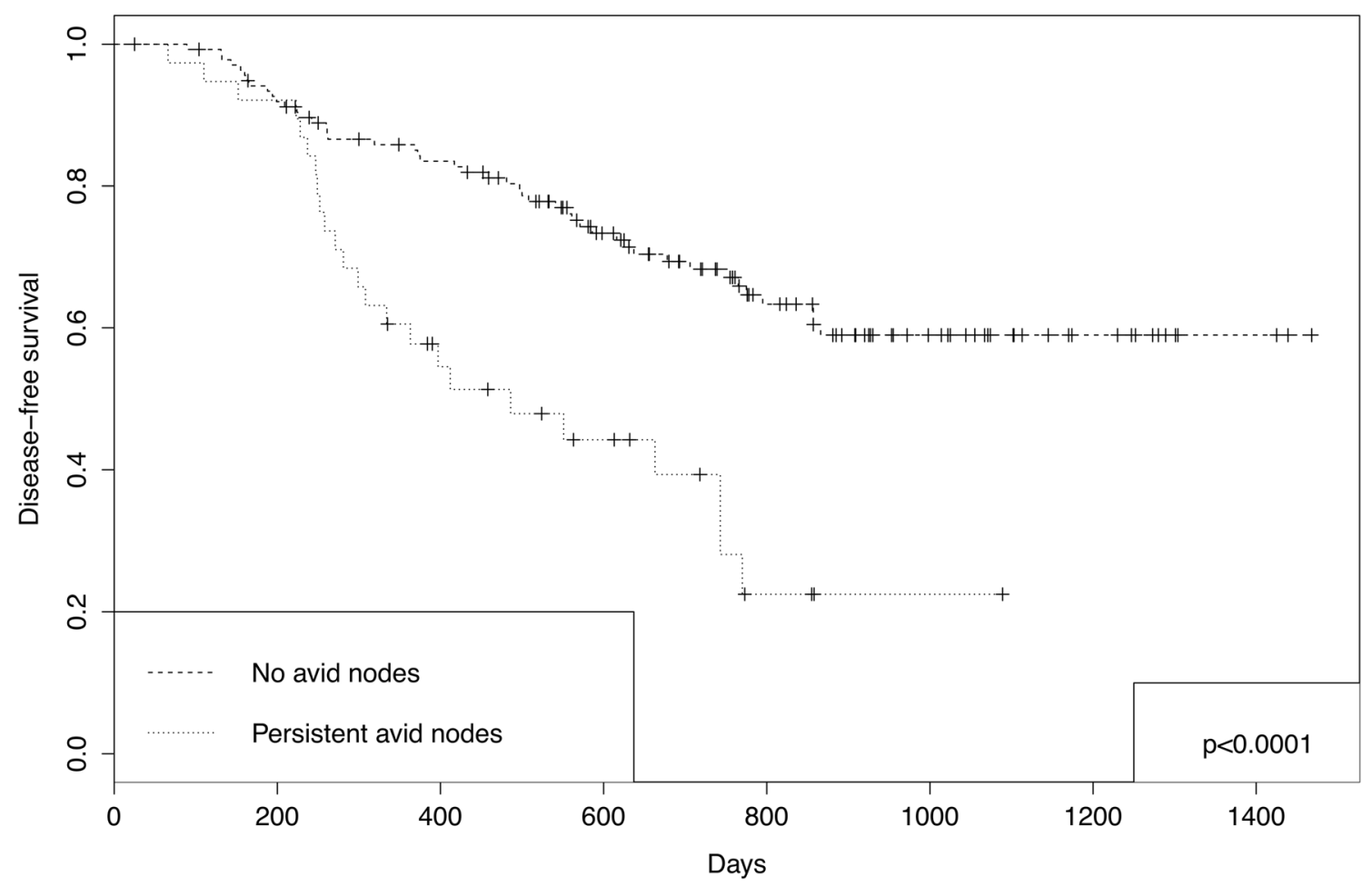

Fig. 1 Disease-free survival and mNR after neoadjuvant chemotherapy; $p$ values generated using log-rank test

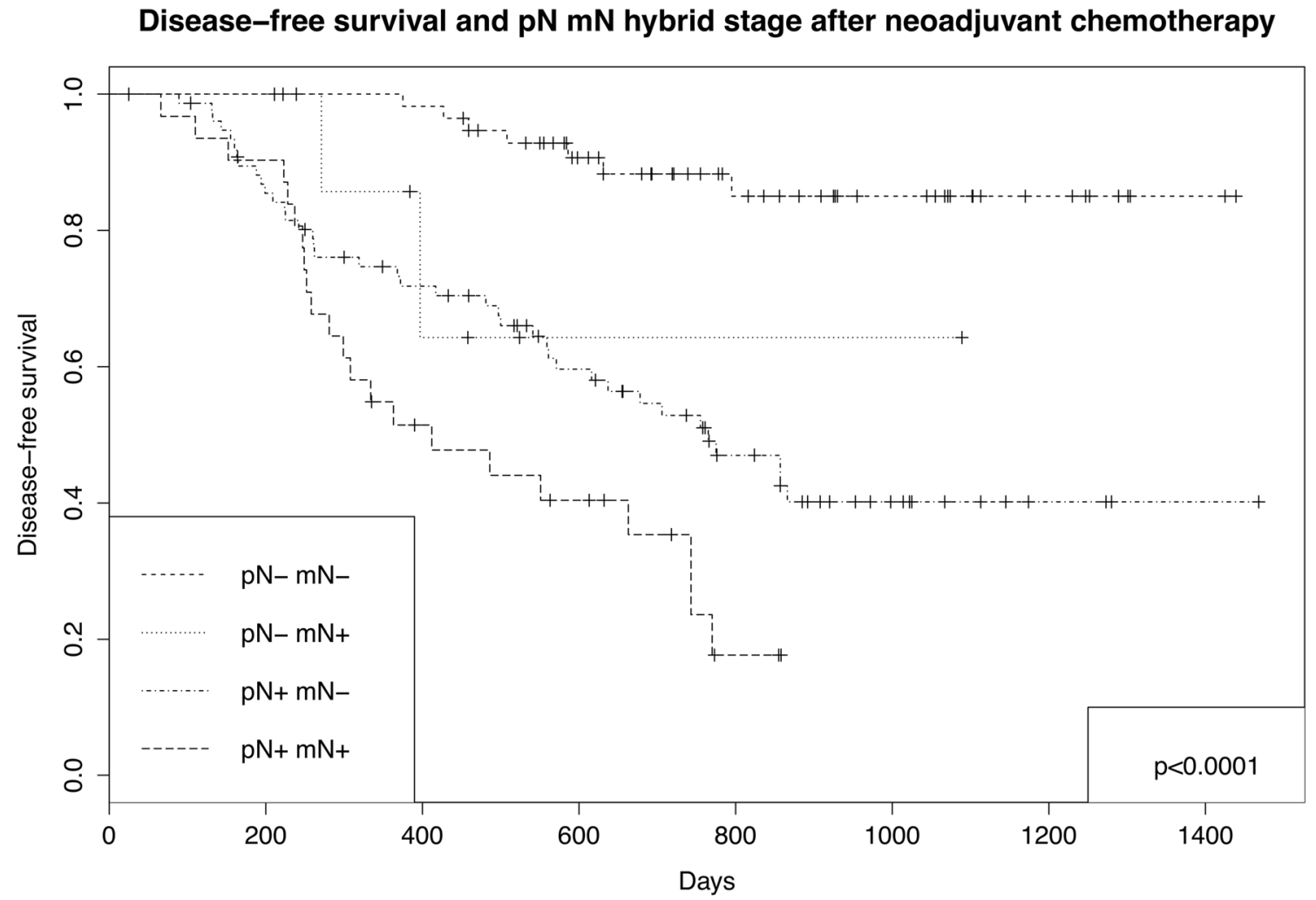

Fig. 2 Disease-free survival $\mathrm{pN} \mathrm{mN}$ hybrid stage after neoadjuvant chemotherapy; $p$ values generated using log-rank test 
Table 4 Predicting early recurrence and death: multivariate binary logistic regression

\begin{tabular}{|c|c|c|}
\hline \multirow[t]{2}{*}{ Characteristic } & \multicolumn{2}{|c|}{ Odds ratio (OR; $95 \%$ confidence interval; $p$ value) } \\
\hline & Within 1 year & Within 2 years \\
\hline \multicolumn{3}{|l|}{$\mathrm{mNR}$} \\
\hline No avid nodes/CMR & Reference & Reference \\
\hline PMR/SMD/PMD & $4.18(1.56-11.2 ; 0.004)$ & $3.63(1.26-10.5 ; 0.017)$ \\
\hline \multicolumn{3}{|l|}{ ypT stage } \\
\hline $1 b$ & Reference & Reference \\
\hline 2 & $0.50(0.45-4.97 ; 0.556)$ & $0.82(0.11-6.01 ; 0.843)$ \\
\hline 3 & $0.30(0.05-2.07 ; 0.217$ & $1.23(0.27-5.50 ; 0.789)$ \\
\hline 4 & $0.47(0.05-4.54 ; 0.514)$ & $5.18(0.58-46.5 ; 0.142)$ \\
\hline \multicolumn{3}{|l|}{ ypN stage } \\
\hline 0 & Reference & Reference \\
\hline 1 & $18.5(1.89-182 ; 0.012)$ & $2.24(0.65-7.77 ; 0.204)$ \\
\hline 2 & $49.7(5.01-493 ;<0.001)$ & $2.93(0.81-10.6 ; 0.101)$ \\
\hline 3 & $74.3(6.66-829 ;<0.001)$ & $4.79(1.14-20.1 ; 0.032)$ \\
\hline \multicolumn{3}{|l|}{ ypL stage } \\
\hline 0 & Reference & Reference \\
\hline 1 & $0.68(0.190-2.43 ; 0.556)$ & $1.54(0.72-3.29 ; 0.332)$ \\
\hline \multicolumn{3}{|l|}{ Resection margin } \\
\hline Clear & Reference & Reference \\
\hline Involved & $1.59(0.54-4.71 ; 0.399)$ & $2.91(0.79-10.7 ; 0.108)$ \\
\hline \multicolumn{3}{|l|}{ pTR } \\
\hline No response & Reference & Reference \\
\hline Response & $0.47(0.09-2.55 ; 0.383)$ & $1.36(0.38-4.91 ; 0.637)$ \\
\hline \multicolumn{3}{|l|}{ mTR } \\
\hline \%Reduction SUVmax & $1.06(0.26-4.36 ; 0.993)$ & $1.06(0.28-4.09 ; 0.931)$ \\
\hline
\end{tabular}

$\mathrm{pN} \mathrm{mN}$ hybrid adjusted for all variables above, other than $\mathrm{ypN}$ and $\mathrm{mNR}$ $\mathrm{pN} \mathrm{mN}$ hybrid

\begin{tabular}{lll}
$\mathrm{pN} 0 \mathrm{mN} 0$ & Reference & Reference \\
$\mathrm{pN}-\mathrm{mN}+$ & $\mathrm{NA}$ & $11.4(0.85-152 ; 0.067)$ \\
$\mathrm{pN}+\mathrm{mN}-$ & $\mathrm{NA}$ & $3.47(1.07-11.3 ; 0.039)$ \\
$\mathrm{pN}+\mathrm{mN}+$ & $\mathrm{NA}$ & $9.19(2.15-39.3 ; 0.003)$ \\
\hline
\end{tabular}

$C M R$, complete metabolic response; $P M R$, partial metabolic response; $S M D$, stable metabolic disease; $P M D$, progressive metabolic disease; $m N R$, metabolic nodal response; $m T R$, metabolic tumor response; $y p T$, pathological tumor stage; $y p L$, pathological venous stage; $p T R$, pathological tumor response micro-environment, perfusion, and hypoxia), at a simplistic level, it may be possible to infer chemo(in)sensitivity. We previously reported primary tumor response on PET-CT (mTR) to be an imperfect surrogate of pathological response (pTR) [17], in agreement with other groups [18], including its successful use within the MUNICON trial [19]. Esophageal cancer comprises a limited number of cancer clones, which vary genomically and probably phenotypically, including their chemosensitivity and propensity to metastasize [20]. Nodal metastases may therefore comprise just one or two selected oligoclones $[21,22]$. We, and other groups, have previously reported dramatic clonal evolution within the primary tumor during the selection pressure of NAC [23, 24], with chemoresistant clones tending to persist or emerge, while chemosensitive clones are lost. Lack of mNR may therefore be an indicator of chemo-resistant clones within resectable lymph nodes, and therefore by extrapolation, a surrogate for the occult distant nodal or hematogenous micrometastases that are responsible for recurrence. This hypothesis is supported by evidence that nodal downstaging, evidenced by imaging in conjunction with $\mathrm{pN}$ stage, represents an independent prognostic marker, likely a surrogate of pathological nodal regression $[25,26]$. Whether this plausibly might be used to guide neoadjuvant and adjuvant therapy is unknown.

While this study provides temporal validation of $\mathrm{mNR}$ as a biomarker, we acknowledge a number of limitations. Firstly, this represents a single-center study with a degree of subjectivity inherent to all imaging studies (although 
one subject to a rigorous MDT process); whether these findings can be generalized to other centers and imaging platforms is not clear. However, similar results have been reported from Japan [27] following largely platinum-based $\mathrm{NAC}$ as a marker of overall survival (although recurrence was not assessed). While we previously found no differences across reconstruction platforms [2], these, and other variables (such as NAC regimen) inevitable of evolving practice, may exert subtle effects we were unable to identify. We were also unable to reliably adjust for adjuvant therapy, and there may be additional factors that we have failed to identify. Secondly, detection biases have been introduced by "on-demand" investigations for recurrence rather than surveillance imaging. Thirdly, while we found consistent direction and sizes of effect for prognosis compared with our development cohort, we saw fewer patients overall with occult unresectable disease resulting in an abandoned resection. This probably reflects improvements in care with time, and hence while the association persisted, its effect size is likely to be less accurate, and requires further validation.

In conclusion, in a cohort of 200 patients immediately consecutive to our discovery cohort, we found lack of a complete mNR (i.e., the development or persistence of avid nodes despite NAC) to again be an independent and seemingly consistent marker of disease progression, recurrence, and death after surgery. This temporal validation provides supportive evidence justifying the assessment of $\mathrm{mNR}$ as a new candidate biomarker for these endpoints in external validation sets. If supported, mNR might represent a new mechanism by which to both risk stratify patients and personalize therapy with esophageal and GEJ cancer.

Funding This study has received funding by the NIHR Oxford Biomedical Research Centre.

\section{Compliance with ethical standards}

Guarantor The scientific guarantor of this publication is John Findlay.

Conflict of interest The authors of this manuscript declare relationships with the following companies:

JMF has a previous unremunerated advisory relationship with Physiomics.

MRM has the following roles to disclose:

Advisory/Consulting Role (payment to the individual) Amgen, BMS, GSK, Merck, Millennium, Physiomics and Roche. Research Funding (payment to the institution) from Amgen, AZ, BMS, Clovis, Eisai, GSK, Immunocore, Johnson \& Johnson, Merck, Millennium, Novartis, Pfizer, Roche and Vertex.

Statistics and biometry One of the authors (JMF) has significant statistical expertise.

Informed consent Written informed consent was waived by the Institutional Review Board.
Ethical approval Institutional Review Board approval was obtained.

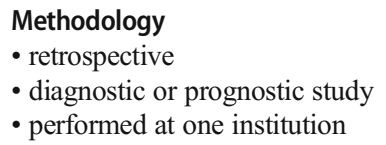

Open Access This article is distributed under the terms of the Creative Commons Attribution 4.0 International License (http:// creativecommons.org/licenses/by/4.0/), which permits unrestricted use, distribution, and reproduction in any medium, provided you give appropriate credit to the original author(s) and the source, provide a link to the Creative Commons license, and indicate if changes were made.

\section{References}

1. Findlay JM, Gillies RS, Franklin JM et al (2016) Restaging oesophageal cancer after neoadjuvant therapy with (18)F-FDG PET-CT: identifying interval metastases and predicting incurable disease at surgery. Eur Radiol 26:3519-3533

2. Findlay JM, Bradley KM, Wang LM et al (2017) Metabolic nodal response as a prognostic marker after neoadjuvant therapy for oesophageal cancer. Br J Surg 104:947

3. Shapiro J, van Lanschot JJB, Hulshof Hulshof MCCM et al (2015) Neoadjuvant chemoradiotherapy plus surgery versus surgery alone for oesophageal or junctional cancer (CROSS): long-term results of a randomised controlled trial. Lancet Oncol 16:1090-1098

4. Davies AR, Gossage JA, Zylstra J et al (2014) Tumor stage after neoadjuvant chemotherapy determines survival after surgery for adenocarcinoma of the esophagus and esophagogastric junction. $\mathrm{J}$ Clin Oncol 32:2983-2990

5. Davies AR, Pillai A, Sinha P et al (2014) Factors associated with early recurrence and death after esophagectomy for cancer. J Surg Oncol 109:459-464

6. Findlay JM, Middleton MR, Tomlinson I (2015) A systematic review and meta-analysis of somatic and germline DNA sequence biomarkers of esophageal cancer survival, therapy response and stage. Ann Oncol 26:624-644

7. Edge SB, Compton CC (2010) The American Joint Committee on Cancer: the 7th edition of the AJCC cancer staging manual and the future of TNM. Ann Surg Oncol 17:1471-1474

8. Wahl RL, Jacene H, Kasamon Y, Lodge MA (2009) From RECIST to PERCIST: evolving considerations for PET response criteria in solid tumors. J Nucl Med 50(Suppl 1):122S-150S

9. Mandard AM, Dalibard F, Mandard JC et al (1994) Pathologic assessment of tumor regression after preoperative chemoradiotherapy of esophageal carcinoma. Clinicopathologic correlations. Cancer 73:2680-2686

10. Medical Research Council Oesophageal Cancer Working Group (2002) Surgical resection with or without preoperative chemotherapy in oesophageal cancer: a randomised controlled trial. Lancet. 359:1727-1733

11. Phase 2 trial of neo-adjuvant oxaliplatin and 5-fluorouracil in oesophageal cancer. EudraCT 2005-001834-34. EU Clinical Trials Register website. https://www.clinicaltrialsregister.eu/ctr-search/ search?query $=$ Adenocarcinoma\&page $=17$. 2006. Accessed December 28, 2016

12. National Cancer Research Institute (2009) A randomised controlled trial comparing standard chemotherapy followed by resection versus ECX chemotherapy followed by resection in patients with resectable adenocarcinoma of the oesophagus. Available via. https:// 
www.ctu.mrc.ac.uk/media/1279/oe05-protocol-version-6-23-dec09.pdf. Accessed May 21, 2019

13. A randomised phase II/III trial of perioperative chemotherapy with or without bevacizumab in operable adenocarcinoma of the stomach and gastro-oesophageal junction. Available via. http://www. isrctn.com/ISRCTN46020948. Accessed May 21, 2019

14. Cunningham D, Allum WH, Stenning SP et al (2006) Perioperative chemotherapy versus surgery alone for resectable gastroesophageal cancer. N Engl J Med 355:11-20

15. R Core Team (2013) R: a language and environment for statistical computing. R Foundation for Statistical Computing, Vienna

16. Allum WH, Blazeby JM, Griffin SM et al (2011) Guidelines for the management of oesophageal and gastric cancer. Gut 60:1449-1472

17. Findlay JM, Bradley KM, Wang LM et al (2017) Predicting pathologic response of esophageal cancer to neoadjuvant chemotherapy: the implications of metabolic nodal response for personalized therapy. J Nucl Med 58:266-275

18. van Rossum PS, Fried DV, Zhang L et al (2016) The incremental value of subjective and quantitative assessment of 18F-FDG PET for the prediction of pathologic complete response to preoperative chemoradiotherapy in esophageal cancer. J Nucl Med 57:691-700

19. Lordick F, Ott K, Krause BJ et al (2007) PET to assess early metabolic response and to guide treatment of adenocarcinoma of the oesophagogastric junction: the MUNICON phase II trial. Lancet Oncol 8:797-805

20. Xing X, Jia S, Wu J et al (2017) Clonality analysis of synchronous gastro-oesophageal junction carcinoma and distal gastric cancer by whole-exome sequencing. J Pathol 243:165-175
21. van Nistelrooij AM, van Marion R, Koppert LB et al (2017) Molecular clonality analysis of esophageal adenocarcinoma by multiregion sequencing of tumor samples. BMC Res Notes 10:144

22. Yuan W, Liu Z, Lei W et al (2017) Mutation landscape and intratumor heterogeneity of two MANECs of the esophagus revealed by multi-region sequencing. Oncotarget 8:69610-69621

23. Findlay JM, Castro-Giner F, Makino S et al (2016) Differential clonal evolution in oesophageal cancers in response to neoadjuvant chemotherapy. Nat Commun 7:11111

24. Murugaesu N, Wilson GA, Birkbak NJ et al (2015) Tracking the genomic evolution of esophageal adenocarcinoma through neoadjuvant chemotherapy. Cancer Discov 5:821831

25. Noble F, Lloyd MA, Turkington R et al (2017) Multicentre cohort study to define and validate pathological assessment of response to neoadjuvant therapy in oesophagogastric adenocarcinoma. $\mathrm{Br} \mathrm{J}$ Surg 104:1816-1828

26. Davies AR, Myoteri D, Zylstra et al (2018) Lymph node regression and survival following neoadjuvant chemotherapy in oesophageal adenocarcinoma. Br J Surg 105(12):1639-1649

27. Miyata H, Yamasaki M, Makino T et al (2016) Impact of number of [(18)F]fluorodeoxyglucose-PET-positive lymph nodes on survival of patients receiving neoadjuvant chemotherapy and surgery for oesophageal cancer. Br J Surg 103:97-104

Publisher's note Springer Nature remains neutral with regard to jurisdictional claims in published maps and institutional affiliations. 